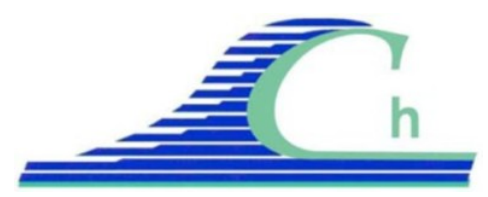

XII ${ }^{\text {ìmes }}$ Journées Nationales Génie Côtier - Génie Civil

Cherbourg, 12-14 juin 2012

DOI:10.5150/jngcgc.2012.036-M @ Editions Paralia CFL

disponible en ligne - http://www.paralia.fr - available online

\title{
Variabilité spatio-temporelle du tarit de côte : plage de Souira Qdima (littoral atlantique marocain)
}

\section{Abdenaim MINOUBI ${ }^{1}$, Mohamed CHAIBI ${ }^{2}$, Emmanuel POIZOT $^{3}$, Mohamed AYT OUGOUGDAL ${ }^{2}$, Yann MEAR ${ }^{3}$, Bendahhou ZOURARAH ${ }^{1}$}

1. Université Chouaib Doukkali, Faculté des Sciences, Laboratoire de Géosciences marines et Sciences du Sol - Unité Associée CNRST (URAC 45), Maroc.

minoubiabdenaim@yahoo.fr

2. Université Cadi Ayyad, Faculté Polydisciplinaire, Département des Sciences Naturelles et de Géographie, Laboratoire GEGEL, Safi, Maroc.

3. Conservatoire national des arts et métiers, Laboratoire Universitaire des Sciences Appliquées de Cherbourg (LUSAC, EA4253, Université de Caen), Cherbourg, France.

\section{Résumé :}

La morphologie des littoraux meubles est complexe et s'organise très souvent sur des secteurs longs de plusieurs kilomètres, où interagissent un grand nombre de facteurs physiques, biologiques et sédimentaires. Dans ces environnements, la tendance naturelle d'érosion est accélérée par des interventions humaines limitant le volume des sédiments essentiel au maintien des plages et à la stabilité du trait de côte (PASKOFF, 1998). Ceci conduit à des perturbations dans les évolutions côtières. Localement, les effets des ouvrages de protection (digues, épis, ouvrages portuaires) contribuent à un déséquilibre de la dynamique sédimentaire littorale. Raison pour laquelle, le recours à la technique de la photo-interprétation, à différentes dates, permet une vision générale et rétrospective des phénomènes. La facilité de traitement et la fiabilité des résultats obtenus amènent un grand nombre de recherches à utiliser les clichés aériens, dont l'analyse diachronique permet de mettre en évidence les transferts sédimentaires.

La plage de Souira Qdima, à l'extrême segment sud du littoral de Safi, constitue une étendue de sable sur la rive droite de l'oued Tensift. Elle est le siège d'un aménagement excessif, qui conduit à une dégradation totale de la dune, par construction d'un grand complexe touristique. Cependant, la rive gauche est une plage sableuse à galets, qui évolue en dehors des actions anthropiques (tendance naturelle).

Afin d'appréhender l'évolution morphologique de ce système sableux à différentes échelles de temps, sur une longue période, nous avons compilé des positions de trait de côte à différentes époques. Cette analyse diachronique du trait de côte, 1954 à 2006, met en évidence un découpage spatial de la plage, en surface d'érosion et d'accrétion, et permet de visualiser l'évolution morphologique dans le temps. Les résultats obtenus montrent une évolution très irrégulière avec des périodes de stabilité, de recul ou d’avancée du rivage. 


\section{Mots clés :}

Tensift - Photo-interprétation - Accrétion - Erosion - Trait de côte - Souira Qdima

\section{Introduction}

Ce travail a pour but de faire progresser la connaissance des milieux côtiers en étudiant l'évolution à long terme des tendances morpho-sédimentaires d'un système côtier anthropisé de la côte atlantique marocaine. Pour atteindre cet objectif, l'interprétation des photographies aériennes et l'analyse de cartes anciennes (SIG) ont été complétées par des relevés sur le terrain ainsi que par des enquêtes auprès des habitants. Ces derniers constituent des sources d'information considérables pour comprendre l'évolution temporelle du trait de côte (GRENIER \& DUBOIS, 1990). Le traitement par photo-interprétation d'une série de compagnes aériennes à différentes dates, donne une vision globale et rétrospective des phénomènes (SABATIER, 2001). C'est un outil adapté à l'analyse quantitative de l'évolution du trait de côte sur de longues périodes et à des échelles compatibles avec les processus étudiés. De nombreux auteurs utilisent les clichés aériens, dont l'analyse diachronique met en évidence les transferts sédimentaires (SUANEZ, 1997 ; DURAND, 1999 ; SABATIER, 2001 ; CHAIBI, 2003 ; LAMBERT, 2006). Comme outil de connaissance, la photo-interprétation est un moyen pour étudier la géomorphologie littorale telle que la genèse des plages, leur pérennité, etc., pour optimiser l'implantation de nouvelles installations et pour suivre et reconstituer l'histoire de ces systèmes côtiers. Dans le domaine de l'urbanisation, l'urbaniste est devenu planificateur et aménageur. Il prépare ainsi l'évolution régionale dans une perspective dynamique, favorisant les tendances naturelles de l'habitat selon un plan d'aménagement. La photographie aérienne peut jouer un rôle important dans la compréhension et la mise en évidence d'un scénario d'évolution des paysages anthropomorphisés. Elle permet aussi aux aménageurs qui ont la responsabilité de façonner le cadre de vie des générations à venir, de confier une attention particulière à l'intégration de la composante historique dans leurs plans de vulnérabilité, de conservation ou d'aménagement et d'estimer les éventuelles modifications morphodynamiques associées, dans la tendance générale d'évolution.

\section{Présentation du site}

La zone d'étude est située dans la baie de Souira Qdima au niveau de l'embouchure de l'oued Tensift (côte ouest marocaine, à $36 \mathrm{~km}$ au Sud de la ville de Safi). La zone est subdivisée en trois compartiments morphologiques qui se distinguent par la présence ou l'absence d'activités humaines : un compartiment médian artificialisé encadré par deux unités sableuses non aménagées au Nord et au Sud (figure 1).

Le compartiment nord correspond au secteur géographique situé entre le port de Souira Qdima et la plage au nord (vers Jorf Ghraba). Cet ensemble morpho-sédimentaire s'étend sur une longueur de 1,6 km. Il est constitué par un cordon dunaire complexe 


\section{XII ${ }^{\text {èmes }}$ Journées Nationales Génie Côtier - Génie Civil \\ Cherbourg, 12-14 juin 2012}

rendu stable par la présence à l'Est d'une grande forêt. A la côte, il est adossé à un estran sableux de 50 à $60 \mathrm{~m}$ de large.

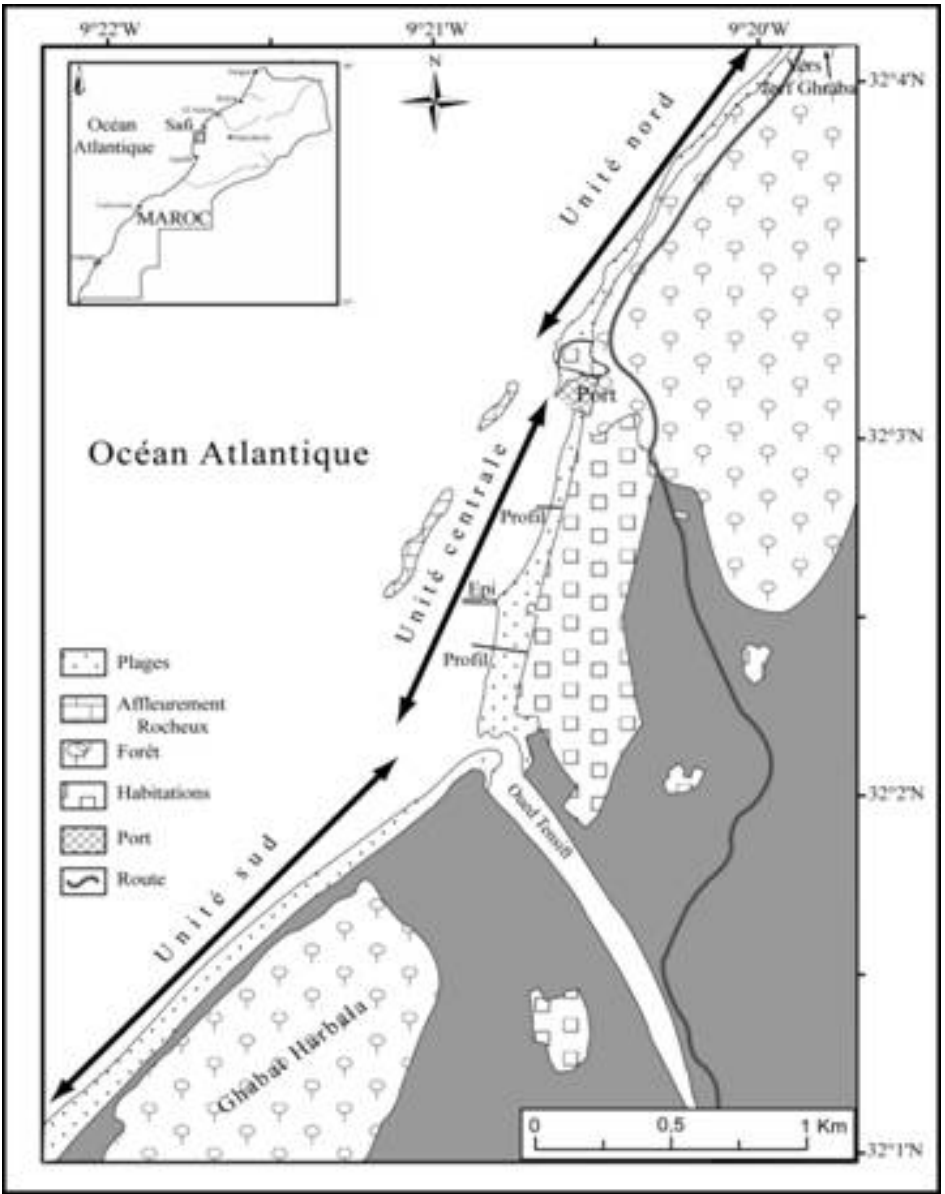

Figure 1. Localisation de la zone d'étude.

Le secteur intermédiaire s'étend du port de Souira Qdima à l'embouchure de l'Oued Tensift. Géomorphologiquement, il comprend la partie aménagée pour les activités humaines (pêche, tourisme, édifices de protections des plages, etc.) (figure 2). De récents aménagements le long du front dunaire ont accentué le déséquilibre des échanges sédimentaires entre le cordon dunaire et la plage proprement dite. La figure 3 donne une illustration d'un profil de plage type de la zone médiane, réalisé par des relevés topographiques à marée basse de vives-eaux.

L'unité méridionale s'étend sur une distance de $1,5 \mathrm{~km}$. Une dune bordière se développe sur la totalité du secteur. Elle est constituée d'un cordon dunaire important, dont la partie orientale est limitée par la forêt de Harbala (Ghabat Harbala). 


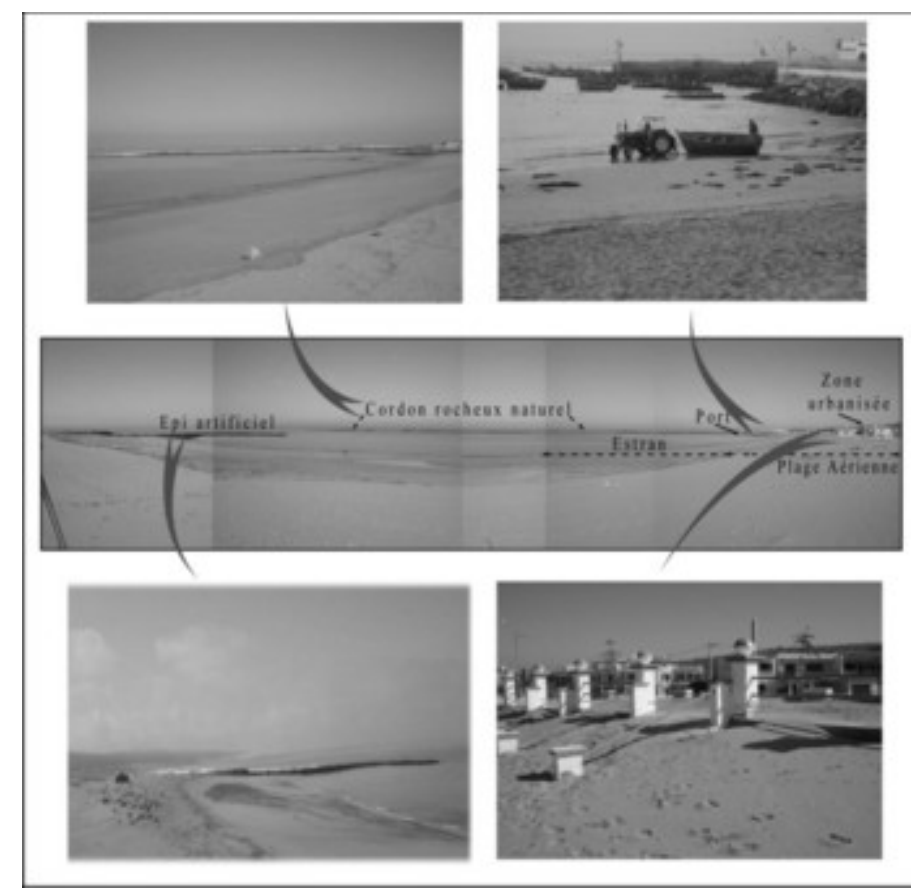

Figure 2. Les différentes composantes de la baie Souira Qdima.

\section{Matériels et méthodes}

De nombreux auteurs assimilent le trait de côte à la ligne de rivage à pleine mer facilement identifiable sur les photographies aériennes. Ainsi, DOUGLAS \& CROWELL (2000) et THIELER \& DANFORTH (1994) prennent en compte la différence radiométrique entre les parties sèches et les parties humides de l'estran. Le trait de côte est aussi défini comme l'ados de plage, i. e. la rupture de pente entre le haut de plage et l'arrière plage. D'autres chercheurs, travaillant sur des littoraux macrotidaux où le marnage est très important, ont choisi le trait de côte comme la limite de la végétation dunaire (ROBIN, 2002). Pour LAMBERT (2006), le trait de côte est défini comme étant la ligne médiane à équidistance des limites des zones de certitude que sont la plage émergée et la mer. Cette approche permet donc le calcul d'une marge d'erreur égale à plus ou moins la demi-valeur de la distance séparant les limites des zones. Des tentatives ont été faites pour proposer des techniques de la délimitation du trait de côte (GRENIER \& DUBOIS, 1990). Cette limite y est facilement détectable du fait du sable humide qui a une couleur plus foncée que le sable sec.

La zone d'étude présente, dans sa partie centrale, des plages aménagées par l'installation d'une corniche balnéaire qui ne nous permet pas de prendre en compte la limite de la végétation comme trait de côte. Les photos aériennes montrent que les missions ont été prises dans des conditions météorologiques (beau temps) et marines (marée) similaires rendant comparable et fiable le trait de côte. Nous avons alors choisi de définir le trait de côte comme la limite entre les parties sèches (plage aérienne) et humides de l'estran. 


\section{XII ${ }^{\text {èmes }}$ Journées Nationales Génie Côtier - Génie Civil \\ Cherbourg, 12-14 juin 2012}

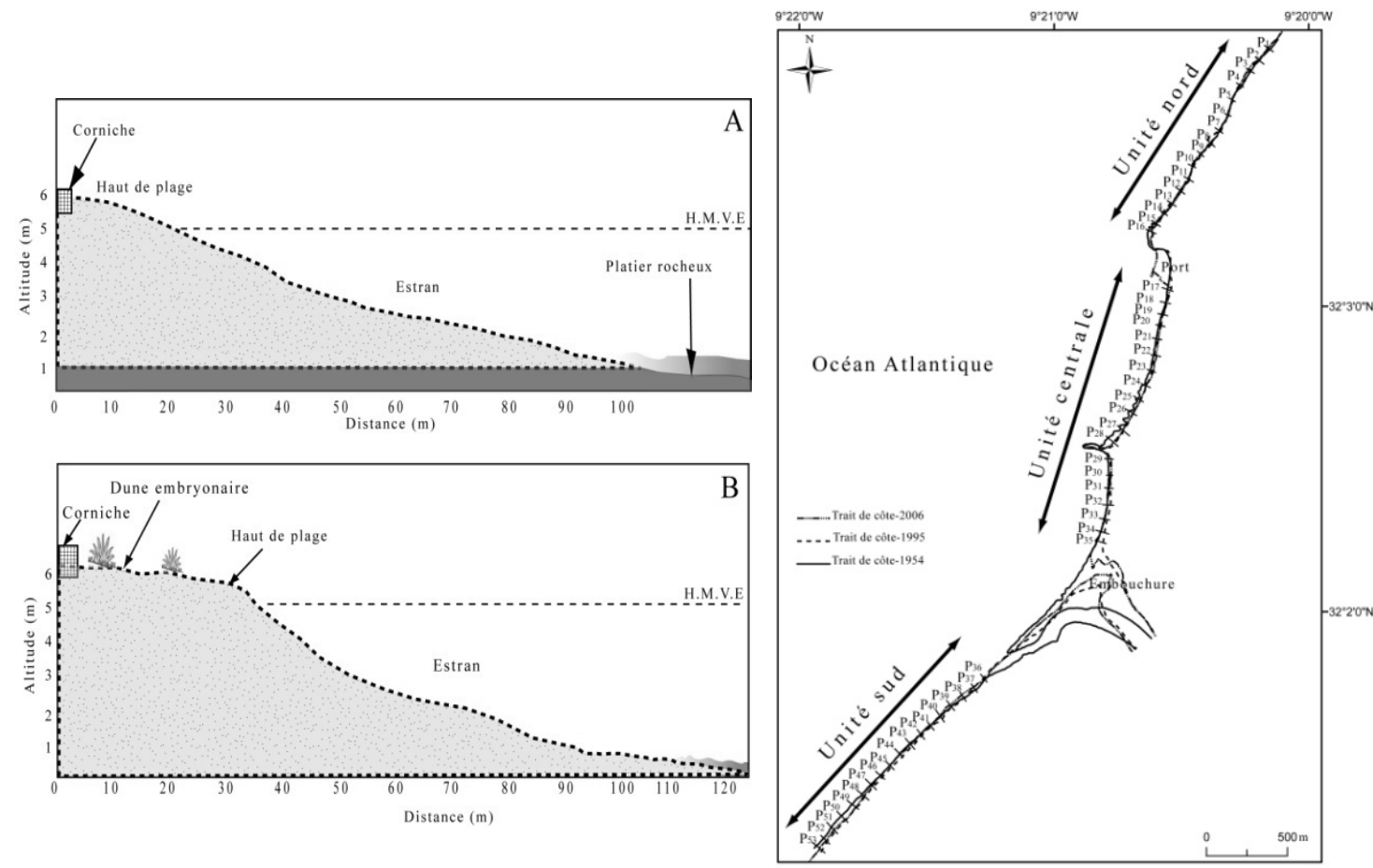

Figure 3 : Profils de plage effectués au nord de l'épi (A) et au sud de l'épi(B) (leurs localisations est sur la figure 1)

Figure 4 : Localisation des transects et évolution des traits de côte entre 1954 et 2006.

Afin de réaliser une étude diachronique du trait de côte à l'aide des missions de photographies aériennes sur une longue période et de quantifier la dynamique spatiotemporelle du trait de côte, nous avons utilisé des campagnes de photographies aériennes de 1954, 1995 et 2006, d'échelle respectivement 1/10000, 1/40000 et 1/30000. Une carte topographique au $1 / 50000$ a été numérisée afin de servir de référence pour le géoréférencement des photographies aériennes.

La marge d'erreur globale doit être définie à partir des incertitudes liées au calage des photos aériennes (repérage des amers) estimées à $\pm 2,5 \mathrm{~m}$, et de l'erreur calculée pour l'ensemble du géoréférencement des amers $( \pm 2 \mathrm{~m})$. Ces incertitudes fixent le seuil à partir duquel on considère qu'il y a une avancée ou un recul du trait de côte. L'erreur maximale des incertitudes est la somme de l'ensemble des erreurs associées aux opérations de prétraitement des données. Elle est, dans notre cas, estimée à $\pm 5,5 \mathrm{~m}$. Les massifs dunaires, dépourvus d'amers repérables ou stables dans le temps, sont responsables de la détérioration locale des valeurs de correction géométrique. La technique photo-interprétation consiste à générer 52 transects équidistants. Ils couvrent l'ensemble des fronts dunaires s'étendant du Jorf Ghraba à l'embouchure de Tensift et sont disposés perpendiculairement au trait de côte (figure 4). L'avancé ou le recul du trait de côte ont été mesurés pour chacun des transects et ont permis d'appréhender 
l'évolution générale de la ligne du rivage. À partir du point d'intersection du trait de côte avec le transect à une date définie, nous obtenons les distances de déplacement du trait de côte.

Afin de faciliter l'analyse, la zone étudiée a été découpée en trois unités morphologiques. Unité septentrionale, située au nord du port (du transect 1 à 16) ; unité médiane entre le port et l'embouchure de Tensift (du transect 17 à 35) et une unité méridionale au sud de l'embouchure (du transect 36 à 53).

\section{Résultats : suivi de la cinématique du trait de côte entre 1954 et 2006}

\subsection{Evolution du trait de côte de 1954 à 1995}

- Unité nord (Jorf Ghraba au Port). La période est caractérisée par une alternance de phases de recul et de progradation de l'estran (figure 5). Une avancé plus significative est marquée autour des transects P1 à P3, alors que du P4 à P8 on constate une légère stabilité de la ligne du rivage, avec des valeurs comprises dans la marge d'erreur. La progression a repris de nouveau son rythme sur les segments situés au niveau de P9 à P10 et de P14 à P15. Les transects P11, P12 et P13 sont caractérisés par une stabilité du trait de côte.

- Unité centrale (entre le port et l'Oued Tensift). Elle se caractérise par une évolution de la ligne du rivage plus distincte par rapport au segment précédent. Au niveau de tous les transects, les tendances montrent une érosion généralisée, caractérisée par une oscillation peu importante (figure 5). Le recul du littoral s'est accentué, au nord de l'épi (P27 et P28).

- Unité sud (au sud de Tensift). L’évolution du rivage se traduit par une progradation entre les transects P38 et P40 avec une avancée importante allant jusqu’à $+1,3$ m/an. La tendance se ralentit vers le Sud (transects 41 et 42) avec une stabilité relative du trait de côte. Entre les transects 43 et 53, une inversion de la tendance de l'évolution caractérisée une érosion qui s'accentue vers le Sud avec une vitesse de recul atteignant 1,15 m/an (transect P53).

\subsection{Evolution du trait de côte de 1995 à 2006}

- Unité nord (Jorf Ghraba au Port). Du Nord au Sud, l'évolution du rivage se traduit par une succession rapide de phases d'érosion et d'engraissement. Si la majorité des transects marque une stabilité du trait de côte certains d'entre eux caractérisent une accrétion (transects 4, 5 et 11) et d'autres une érosion (transects 14 et 15).

- Unité centrale (entre Port et Tensift). Le secteur s'étend du port, à l'embouchure de Tensift et présente une alternance de couloir de recul et de progression. Des portions du rivage sont marquées par un engraissement, (transects 17, 30 et 31). A l'inverse, les transects 21 à 23 caractérisent une zone en érosion. Les profils P23 à P35 


\section{XII ${ }^{\text {èmes }}$ Journées Nationales Génie Côtier - Génie Civil \\ Cherbourg, 12-14 juin 2012}

indiquent une progression généralisée accélérée du trait de côte avec un rythme de progression estimé entre $+0,8$ et $+5,2 \mathrm{~m} / \mathrm{an}$.

- Unité sud (au sud de Tensift). Au niveau des transects P36 et P37, le recul du littoral atteint une vitesse importante variant de $-0,8$ à - $1 \mathrm{~m} / \mathrm{an}$. Cependant, à partir du transect suivant (P38) le compartiment est caractérisé par une avancée généralisée du trait de côte. les tendances évolutives sont inversées, avec une progradation qui peut atteindre jusqu'à $+2 \mathrm{~m} / \mathrm{an}$ entre P39 et P41. Cette situation se poursuit au sud du secteur avec néanmoins un léger ralentissement du taux d'aggradation (figure 5).
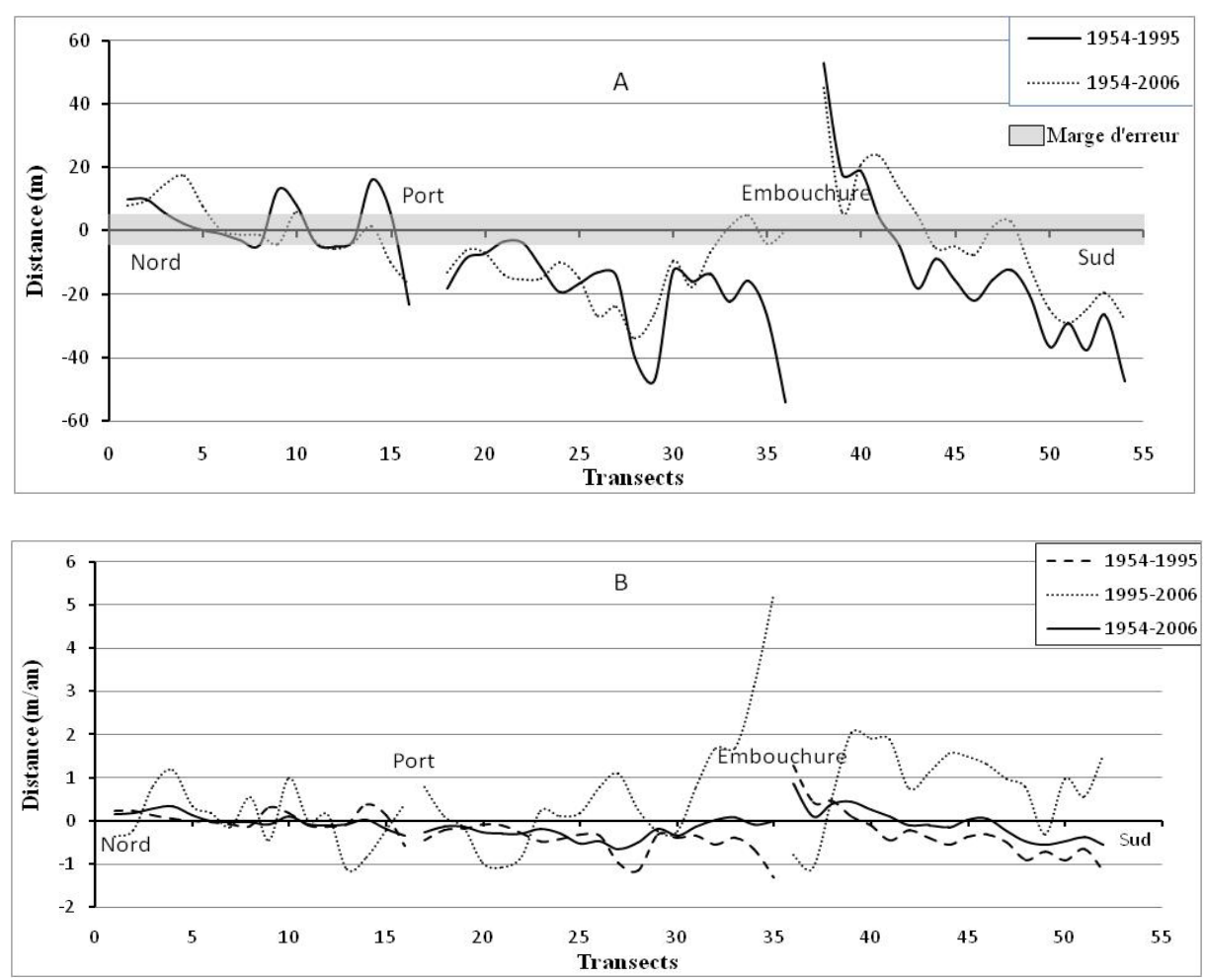

Figure 5. Evolution (en $m$ ) du rivage de Jorf Ghraba au Nord jusqu'au sud de l'Oued Tensift au Sud entre 1954 et 2006 : A- évolution cumulée, B- évolution moyenne annuelle.

\section{Conclusion}

L'évolution spatiale et temporelle du littoral atlantique de la côte marocaine n'est pas homogène. Les résultats obtenus sur la baie de Souira Qdima, pour la période 19542006 le confirme. La zone septentrionale, située entre Jorf Ghraba et le port de Souira Qdima connaît une alternance de phases de recul, de progression et de stabilité. Le secteur central est le plus touché par le processus d'érosion du rivage avec un taux moyen de recul de -20 m, généralisé pendant la période 1954-1995 (avant les aménagements). Cette tendance négative a été renversée dans certaines portions de cette région au cours de la période 1995-2006. L’action anthropique se traduit par une avancé 
du trait de côte. La construction d'une corniche a joué un rôle important en favorisant l'engraissement de la plage.

Dans le secteur méridional, la période d'étude est caractérisée, comme pour la partie nord, par une alternance de phases de recul, de progression et de stabilité de l'estran. Cette partie de la côte devient de plus en plus vulnérable aux actions marines.

\section{Remerciements :}

Ce travail a été réalisé dans le cadre de l'action intégré Franco-Marocaine $\mathrm{n}^{\circ}$ MA/07/179, entre la Faculté Polydisciplinaire de Safi et l’Institut National des Sciences et techniques de la mer (Cherbourg) du Conservatoire national des arts et métiers (France).

\section{Références}

CHAIBI M. (2003). Dynamique sédimentaire et morphogenèse actuelle du littoral d'El Jadida, Maroc. Thèse, Université d'Aix-Marseille I, France, 236 p.

DOUGLAS B.C., CROWELL M. (2000). Long term shoreline prediction and error propagation. Journal of Coastal Research, Vol. 16, 1, pp 145-152.

DURAND P. (1999). L'évolution des plages de l'ouest du Golf du Lion au XXème siècle. Cinématique du trait de côte, dynamique sédimentaire, analyse prévisionnelle. Thèse, Université Lumière Lyon II, Lyon, 460 p.

GRENIER A., DUBOIS J.M.M. (1990). Évolution littorale récente par télédétection : synthèse méthodologique. Photo-interprétation, n 1990/6, pp 3-16.

LAMBERT A. (2006). Protection durable du littoral Varois: fonctionnement hydrosédimentaire de plages microtidales équipées de systèmes de drainage. Thèse, Université Aix-Marseille I, 377 p.

PASKOFF R. (1998). Le littoral du Parc Naturel Régional de la Camargue après la grande tempête de décembre 1997. Parc Naturel Régional de Camargue, Arles.

ROBIN M. (2002). Télédétection et modélisation du trait de côte et de sa cinématique. Le littoral regards, pratiques et savoirs. Edition Ens, pp 95-116.

SABATIER F. (2001). Fonctionnement et dynamique morphosédimentaire du littoral du delta du Rhône. Thèse, Université Aix-Marseille III, France, 285 p.

SUANEZ S. (1997). Dynamiques sédimentaires actuelles et récentes de la frange littorale orientale du delta du Rhône. Thèse, Université de Provence, Aix-en-Provence, $282 \mathrm{p}$.

THIELER E.R., DANFORTH W.W. (1994). Historical shoreline mapping (I): improving techniques and reducing positioning errors. Journal of Coastal Research, Vol. 10, 3, pp 549-563. 